\title{
NMR Spin-Lattice Relaxation and Tunnelling of Partially Deuterated Methyl Groups
}

\author{
H. Langen* and W. Müller-Warmuth \\ Institut für Physikalische Chemie der Westfälischen Wilhelms-Universität Münster, Münster
}

Z. Naturforsch 46a, 1123-1130 (1991); received November 5, 1991

\begin{abstract}
Proton spin lattice relaxation rates have been measured at 15 and $30 \mathrm{MHz}$ and down to $5 \mathrm{~K}$ for the partially deuterated molecular crystals 4 -F-toluene, 4-Cl-toluene, and 2,6- $\mathrm{Cl}_{2}$-toluene. The behaviour of these materials is governed by methyl group tunnelling. As compared with the undeuterated compounds, the low temperature relaxation is enhanced and the details depend on the removal of the symmetry coupling between rotator and spin states. The hindering barriers remain unchanged, the $\mathrm{A}$ to $\mathrm{E}$ conversion rates are faster, and relaxation is dominated by spectral density contributions $J\left(\omega_{0}\right)$ and $J\left(2 \omega_{0}\right)$. In one case an additional influence of level-crossing energy transfer on relaxation is observed. Field-cycling spectroscopy reveals steps rather than peaks if the proton spin Zeeman and tunnelling splittings match.
\end{abstract}

\section{Introduction}

The rotation of methyl groups in solids as the simplest example of molecular motion has been extensively studied for many years both experimentally and theoretically. In particular, quantum mechanical tunnelling at low temperatures, in addition to thermally activated random reorientation at elevated temperatures, has been the subject of numerous papers by various authors [1]. The quantum effect manifests itself as a splitting of the torsional ground state, which can be directly measured by inelastic neutron scattering if the hindering barrier is low enough [2]. Further on, NMR methods have contributed substantially to obtain information about the tunnelling mechanism, and here in particular studies of the frequency and temperature dependence of the spin-lattice relaxation time $T_{1}[3]$.

The results, including those of the relaxation, depend on the symmetry properties of the $\mathrm{CH}_{3}$ group. Each rotator state is coupled to a set of spin states of the same symmetry to satisfy the Pauli exclusion principle and the symmetry requirement of the total wavefunction. We have therefore an $A$ spin state with a total spin $3 / 2$ combined with the $A$ rotator state, and $E_{\mathrm{a}, \mathrm{b}}$ spin states (total spin 1/2) combined with $E_{\mathrm{b}}$ and $E_{\mathrm{a}}$ rotator states, respectively [4]. For asymmetric $\mathrm{CH}_{2} \mathrm{D}$ and $\mathrm{CHD}_{2}$ groups this symmetry coupling

\footnotetext{
* Jetzt: Physikalisches Institut der Universität Stuttgart. Reprint requests to Prof. Dr. W. Müller-Warmuth, Institut für Physikalische Chemie, Universität Münster, W-4400 Münster.
}

does no longer exist. The rotator states are of course also tunnel split, but to a lesser extent, since the moment of inertia is a factor $4 / 3$ or $5 / 3$ larger than that of $\mathrm{CH}_{3}$. Most important, however, is the influence of the absence of the correlation between spin and rotator states on the relaxation.

As far as we know, this effect has not yet been studied in detail, although it has been mentioned repeatedly by various authors or applied to thermodynamic or optical studies [1,5-10]. Previous $T_{1}$ measurements on partially deuterated alkanes were confined to the classical regime since the hindering barriers were large [11]. With the intent to investigate the effect of partial deuteration of the methyl group on the proton spin-lattice relaxation over larger temperature ranges some substituted toluenes with barrier heights between 1.9 and $3.3 \mathrm{~kJ} / \mathrm{mol}$ were selected. These materials are known to display quantum phenomena leading to anomalous relaxation [12].

\section{Experimental Details}

4-fluoro-, 4-chloro- and 2,6-dichloro-toluene were purchased from Aldrich or Merck companies, respectively, and had a chemical purity better than $99 \%$. Partially deuterated compounds $4-\mathrm{F}-\mathrm{C}_{6} \mathrm{H}_{4} \mathrm{CH}_{2} \mathrm{D}$, 4-F- $\mathrm{C}_{6} \mathrm{H}_{4} \mathrm{CHD}_{2}$, 4-Cl- $\mathrm{C}_{6} \mathrm{H}_{4} \mathrm{CH}_{2} \mathrm{D}$, and 2,6- $\mathrm{Cl}_{2}$ $\mathrm{C}_{6} \mathrm{H}_{3} \mathrm{CH}_{3} \mathrm{D}$ were synthesized by reducing the corresponding benzyl halides or benzoic acids. $\mathrm{LiAlH}_{4}$ or LiAlD $_{4}$ in tetrahydrofuran was used as a reducing agent. The degree of deuteration appeared to be better than $96 \%$. From all the materials oxygen was re- 
moved as usual by several freeze-pump-thaw cycles before sealing the sample.

The spin-lattice relaxation time $T_{1}$ was measured in the same way as outlined in previous papers [3] by $90^{\circ}-\tau-90^{\circ}$ pulse sequences and evaluating the initial slope of the relaxation function. ${ }^{1} \mathrm{H}$ NMR frequencies of $\omega_{0} / 2 \pi=15 \mathrm{MHz}$ and $30 \mathrm{MHz}$ were applied. Accuracy considerations of earlier $T_{1}$ measurements hold as well; the error is of the order of $\pm 10 \%$. Level-crossing spectroscopy was applied to $2,6-\mathrm{Cl}_{2}-\mathrm{C}_{6} \mathrm{H}_{3} \mathrm{CH}_{2} \mathrm{D}$ using the same experimental system as previously $[3$, 12]. Relaxation peaks were searched for that occur when the Zeeman and tunnel splitting match. Temperature was controlled by continuous flow liquid helium cryostats and independently measured by $\mathrm{NiCr} / \mathrm{AuFe}$ thermocouples.

\section{Results}

Figure 1 shows the relaxation rates as functions of the reciprocal temperature as obtained for 4-fluorotoluene and its partially deuterated derivatives. As may be realized from the figures, the slopes of these presentations remain more or less unchanged at the high temperature side, whereas the frequency-independent maximum does no longer appear for the partially deuterated compounds. The latter, and in particular the $\mathrm{CH}_{2}$ D-compound, exhibit clearly stronger relaxation at very low temperatures, and there is a curvature in the slopes.

$1 / T_{1}$ results for the comparison of the 4-chlorotoluenes $\mathrm{C}_{6} \mathrm{H}_{4} \mathrm{ClCH}_{3}$ and $\mathrm{C}_{6} \mathrm{H}_{4} \mathrm{ClCH}_{2} \mathrm{D}$ are plotted in Figure 2. At first sight the differences look less striking, but again the (small) first maximum disappears upon partial deuteration and at the low temperature side the relaxation is stronger.

The 2,6-dichloro-toluene compounds (Fig. 3) are distinguished by a still higher barrier hindering the methyl group rotation. The deviations from a classical behaviour are less stringent and may be recognized more clearly for $\mathrm{CH}_{3}$ rather than for $\mathrm{CH}_{2} \mathrm{D}$. Here low temperature relaxation is not stronger for the partially deuterated material.

For the partially deuterated fluoro- and chlorotoluenes no field-cycling experiments to determine the tunnelling frequency were possible, since the low temperature relaxation times were too short. The fieldcycling spectrum of 2,6- $\mathrm{Cl}_{2}-\mathrm{C}_{6} \mathrm{H}_{3} \mathrm{CH}_{2} \mathrm{D}$ (Fig. 4) shows steps rather than peaks at field values which differ by a factor of 2 from one another. Curve 1 was registered starting from a reference field of $0.35 \mathrm{~T}$, curve 2 starting from $0.7 \mathrm{~T}$.

\section{Interpretation}

\section{1. $\mathrm{CH}_{3}$ Relaxation}

The relaxation rates of the undeuterated materials (Figs. 1-3, top) are only presented for the purpose of comparison. They can be interpreted by Haupt's equation

$$
\begin{aligned}
& 1 / T_{1}=C_{1} \sum_{n=-2}^{+2} \frac{n^{2} \tau_{\mathrm{c}}}{1+}+\left(\omega_{\mathrm{t}}+n \omega_{0}\right)^{2} \tau_{\mathrm{c}}^{2} \\
&+C_{2} \sum_{n=1}^{2} \frac{n^{2} \tau_{\mathrm{c}}}{1+n^{2} \omega_{0}^{2} \tau_{\mathrm{c}}^{2}},
\end{aligned}
$$

as already successfully verified for various molecular crystals in many previous papers [3, 12]. The relaxation strength $C_{1}$ accounts for dipole-dipole interactions which are connected with a change of symmetry of the $\mathrm{CH}_{3}$ rotator from A to E. $C_{2}$ takes notice of transitions without change of symmetry, which are only allowed for intermolecular interactions of the methyl group.

The full curves correspond to curve fittings where the correlation time was approximated by

$$
\frac{1}{\tau_{\mathrm{c}}}=\frac{1}{\tau_{\mathrm{c}}^{\prime}}+\frac{1}{\tau_{\mathrm{c}}^{\prime \prime}} .
$$

Both correlation times follow an Arrhenius law with apparent activation energies $E_{\mathrm{A}}^{\prime}$ and $E_{\mathrm{A}}^{\prime \prime}$. The limiting values $\tau_{\mathrm{c}}^{\prime}\left(E_{\mathrm{A}}^{\prime}\right)$ and $\tau_{\mathrm{c}}^{\prime \prime}\left(E_{\mathrm{A}}^{\prime \prime}\right)$ are correlation times for the classical random reorientation and the low temperature process in question, respectively. $E_{\mathrm{A}}^{\prime}$ can be identified with the classical activation energy $E_{\mathrm{A}}$ corresponding to the height of the hindering barrier. For low barriers, $E_{\mathrm{A}}^{\prime \prime}$ agrees in many cases with the splitting $E_{01}$ between the torsional ground and the first excited state. This holds for 2,6-dichloro-toluene, but other materials are exceptions. Table 1 , which collects the various parameters and also the tunnel splittings and the energies of the torsional excitations [12,13], shows that 4-Cl-toluene (and likewise 4-Br- and 4-I-toluene) exhibits in some respects a different relaxation behaviour. But this is not the topic of the present paper and will be discussed elsewhere [14]. 

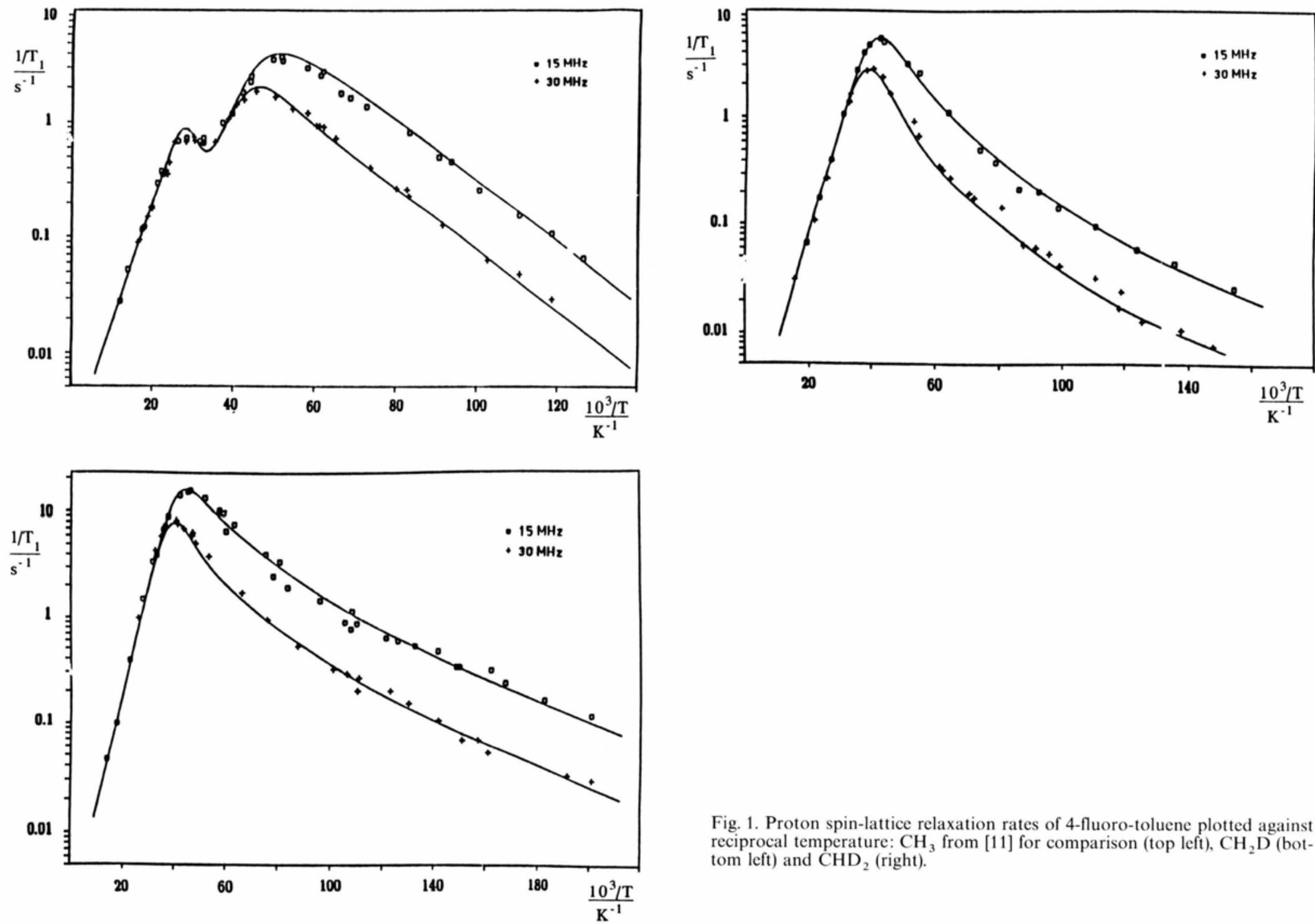

Fig. 1. Proton spin-lattice relaxation rates of 4-fluoro-toluene plotted against reciprocal temperature: $\mathrm{CH}_{3}$ from [11] for comparison (top left), $\mathrm{CH}_{2} \mathrm{D}$ (bottom left) and $\mathrm{CHD}_{2}$ (right). 

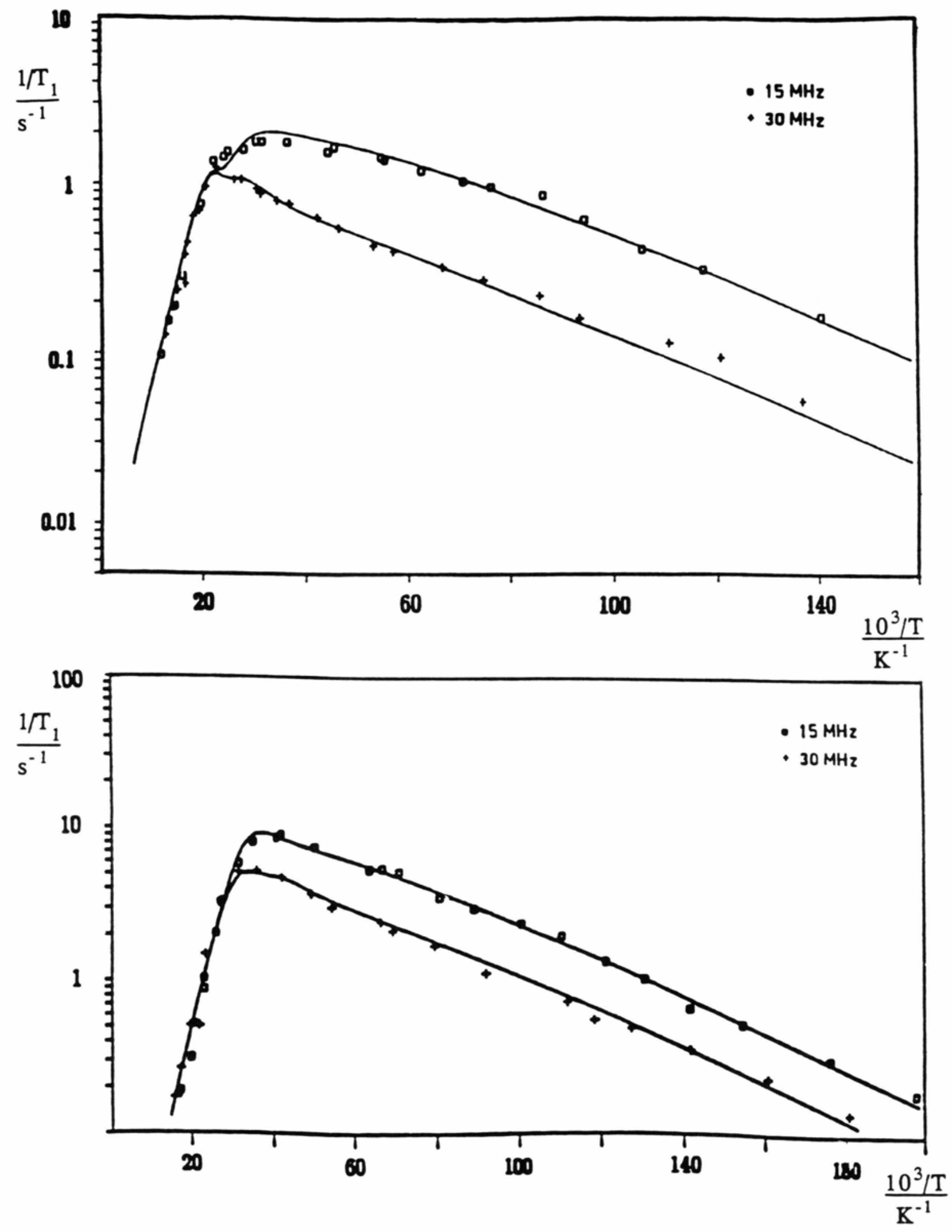

Fig. 2. Proton spin-lattice relaxation rates of 4-chloro-toluene plotted against reciprocal temperature: $\mathrm{CH}_{3}$ (top) and $\mathrm{CH}_{2} \mathrm{D}$ (bottom). 

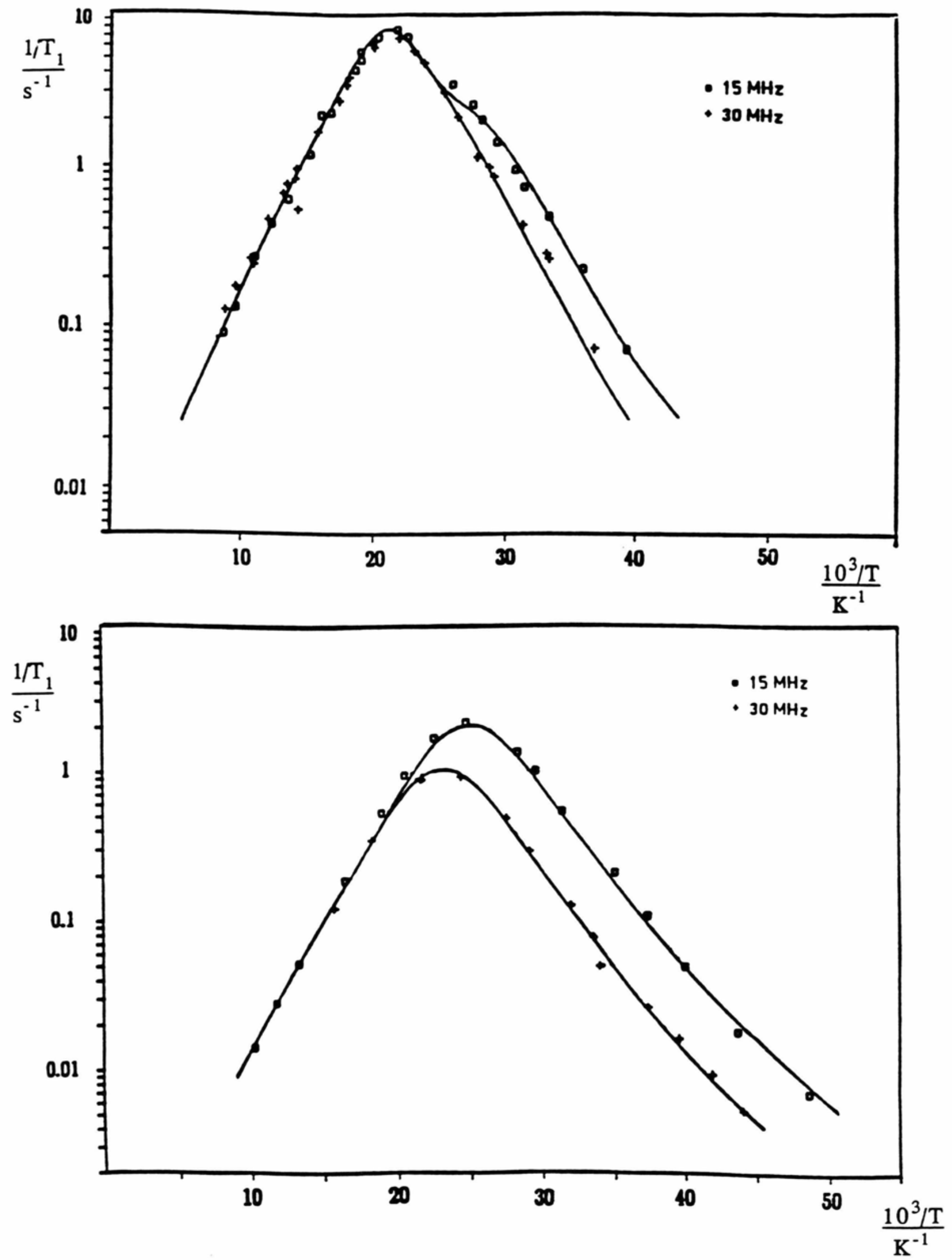

Fig. 3. Proton spin-lattice relaxation rates of 2,6-dichloro-toluene plotted against reciprocal temperature: $\mathrm{CH}_{3}$ (top) and $\mathrm{CH}, \mathrm{D}$ (bottom). 
Table 1. Numerical results for the proton spin-lattice relaxation and rotational tunnelling of $\mathrm{CH}_{3}$ groups. Tunnel splittings of the torsional ground state $\left(\omega_{t}^{0}\right)$ and torsional excitations $\left(E_{01}\right)$ were obtained from inelastic neutron scattering [12, 13].

\begin{tabular}{lccccccccccc}
\hline & $\frac{\tau_{\mathrm{c}}^{\prime}}{10^{-13} \mathrm{~s}}$ & $\frac{E_{\mathrm{A}}^{\prime}}{\mathrm{kJ} / \mathrm{mol}}$ & $\frac{\tau_{\mathrm{c}}^{\prime \prime}}{10^{-10} \mathrm{~s}}$ & $\frac{\mathrm{E}_{\mathrm{A}}^{\prime \prime}}{\mathrm{kJ} / \mathrm{mol}}$ & $\frac{C_{1}}{10^{9} \mathrm{~s}^{-2}}$ & $\frac{C_{2}}{10^{9} \mathrm{~s}^{-2}}$ & $\frac{h \omega_{\mathrm{t}}^{0}}{\mu \mathrm{eV}}$ & $\frac{E_{01}}{\mathrm{~kJ} / \mathrm{mol}}$ \\
\hline 4-F-toluene & 1.3 & 1.9 & 6.1 & 0.48 & 2.2 & 0.31 & 17.6 \\
4-Cl-toluene & 30 & 2.3 & 30 & 0.29 & 0.09 & 0.15 & 1.22 \\
2.6- $\mathrm{Cl}_{2}$-toluene & 1.4 & 3.3 & 9.0 & 1.4 & 3.0 & 0.12 & 1.26 \\
\hline
\end{tabular}

Table 2. Numerical results for the $\mathrm{CH}_{2} \mathrm{D}$ and $\mathrm{CHD}_{2}$ proton relaxation and tunnelling. Details are explained in the text.

\begin{tabular}{|c|c|c|c|c|c|c|c|c|}
\hline & $\frac{\tau_{\mathrm{c}}^{\prime}}{10^{-13} \mathrm{~s}}$ & $\frac{E_{\mathrm{A}}^{\prime}}{\mathrm{kJ} / \mathrm{mol}}$ & $\frac{\tau_{c}^{\prime \prime}}{10^{-10} \mathrm{~s}}$ & $\frac{\mathrm{E}_{\mathrm{A}}^{\prime \prime}}{\mathrm{kJ} / \mathrm{mol}}$ & $\frac{\tau_{\mathrm{c}}^{\prime \prime \prime}}{10^{-8} \mathrm{~s}}$ & $\frac{E_{\mathrm{A}}^{\prime \prime \prime}}{\mathrm{kJ} / \mathrm{mol}}$ & $\frac{C}{10^{9} \mathrm{~s}^{-2}}$ & $\frac{C_{1}}{10^{9} \mathrm{~s}^{-2}}$ \\
\hline 4-F-toluene- $\mathrm{CH}_{2} \mathrm{D}$ & 4.3 & 2.0 & 15 & 0.50 & 3 & 0.18 & 1.5 & - \\
\hline 4-F-toluene-CHD̃ 2 & 3.8 & 2.0 & 11 & 0.59 & 9 & 0.20 & 0.46 & - \\
\hline 4-Cl-toluene- $\mathrm{CH}_{2} \mathrm{D}$ & 6.0 & 2.3 & 36 & 0.23 & & & 0.72 & 0.06 \\
\hline 2.6- $\mathrm{Cl}_{2}$-toluene- $\mathrm{CH}_{2} \mathrm{D}$ & 4.0 & 3.3 & 30 & 1.7 & - & - & 1.4 & - \\
\hline
\end{tabular}

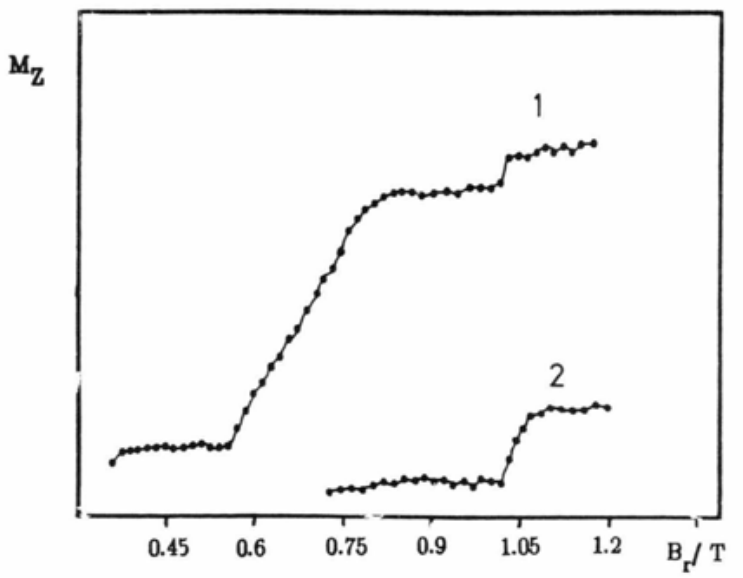

Fig. 4. Field-cycling spectrum of 2,6-dichloro-toluene- $\mathrm{CH}_{2} \mathrm{D}$ at $10 \mathrm{~K}$. The $z$-component of the proton magnetization $M_{z}^{2}$ in arbitrary units is plotted versus the relaxation magnetic field $B_{r}$, which is varied in steps of $0.01 \mathrm{~T}$.

\section{2. $\mathrm{CH}_{2} \mathrm{D}$ Relaxation}

Partial deuteration leads (in addition to the smaller tunnel splitting) to a removal of the symmetry coupling between rotator and spin states. Intra-methyl relaxation is therefore no longer necessarily connected with A to $E_{\mathrm{a}}, E_{\mathrm{b}}$ tunnel transitions, but may be effective at frequencies $\omega_{0}$ and $2 \omega_{0}$, if the rotator system provides via its coupling to the phonons spectral densities $J\left(\omega_{0}\right)$ and $J\left(2 \omega_{0}\right)$ at these frequencies. The experimental results of Figs. $1-3$ can essentially be described by the second series of terms of (1) only, where $C_{2}$ has now of course another meaning and magnitude since it includes the importance of relaxation due to the $\mathrm{H}-\mathrm{H}$-coupling within a $\mathrm{CH}_{2} \mathrm{D}$ group. We will therefore use the symbol $C$ rather than $C_{2}$, cf. Table 2 .

The curve fittings of Fig. 1 (bottom left) and Fig. 3 (right) are based on the second part of (1) only. The correlation times are composed of two (cf. (2)) or even three contributions as already predicted by Zweers and Brom [6]. The model parameters are listed in Table 2.

Most remarkable are the relaxation rates of $4-\mathrm{Cl}$ toluene- $\mathrm{CH}_{2}$ D, Fig. 2, bottom, which are clearly enhanced as compared with those of the $\mathrm{CH}_{3}$ compound. Fitting is again possible by essentially considering the spectral densities $J\left(\omega_{0}\right)$ and $J\left(2 \omega_{0}\right)$, but some small contributions of resonance transfer at $\omega_{0}=\omega_{\mathrm{t}}$ and $2 \omega_{0}=\omega_{\text {t }}$ have to be taken into account in addition. The importance of this effect for $\mathrm{CH}_{2} \mathrm{D}$ relaxation was outlined by Svare and Tunstall [5]. The curve fitting of Fig. 2 considers this by including also the first series of terms of (1), but with a much smaller value for $C_{1}$.

\section{3. $\mathrm{CHD}_{2}$ Relaxation}

Apart from the reduced relaxation strength, the behaviour of $4-\mathrm{F}-\mathrm{C}_{6} \mathrm{H}_{4} \mathrm{CHD}_{2}$ (Fig. 1, right) does not look too much different from that of $4-\mathrm{F}-\mathrm{C}_{6} \mathrm{H}_{4} \mathrm{CH}_{2} \mathrm{D}$ (Fig. 1, centre). There is no longer an intramolecular 
contribution due to the dipole-dipole interaction within the methyl group, and the intermolecular part (interaction with other protons) is governed by the same rules as that for the $\mathrm{CH}_{2} \mathrm{D}$ compound. The differences between the low temperature correlation times (Table 2) are due to the changes of the rotator levels upon isotope substitution. As in 4.2, the small $I=1$ spin of $\mathrm{D}$ may be neglected.

\subsection{Field-cycling Spectrum}

In the preparation stage of this experiment the tunnel system is in thermal equilibrium with the lattice, and the spin system is hot after application of saturation pulses. If the relaxation field is then swept until the proton Zeeman splitting and the tunnel splitting match, resonant energy is transferred from the proton spin to the colder tunnel system. The proton magnetization recovers and a level-crossing peak appears, when $M_{z}$ is monitored by an increase of $T_{1}^{-1}$. For $\mathrm{CH}_{3}$ groups, due to the symmetry requirements the tunnel system is easily saturated, since the A to E conversion rate is low. The features of the resonance peak are greatly predetermined by this [15].

For asymmetric $\mathrm{CH}_{2} \mathrm{D}$ groups the conversion rate is fast because the lattice phonons do not need the nuclear dipolar interaction to make the rotational transition [9]. The levels become lifetime broadened and the tunnel system remains unsaturated during the experiment. Figure 4 shows that steps occur rather than peaks when the level-crossing conditions are met. We believe that the two steps belong to $\omega_{0}=\omega_{1}^{0} / 2$ and $\omega_{0}=\omega_{\mathrm{t}}^{0}$, where the tunnel frequency can be determined to be roughly $\omega_{\mathrm{t}}^{0} / 2 \pi=48 \mathrm{MHz}$.

\section{Discussion and Conclusions}

Inspection of the results and their interpretation reveals a number of observations, which are of general importance:

(i) The barrier heights $\left(E_{\mathrm{A}}^{\prime}=E_{\mathrm{A}}\right)$ remain more or less unchanged upon partial deuteration of the methyl groups.

(ii) In contrast to the classical regime, if tunneling plays a role, the low temperature relaxation rates appear to be enhanced rather than reduced as compared with $\mathrm{CH}_{3}$. (iii) Relaxation is dominated by spectral density contributions $J\left(\omega_{0}\right)$ and $J\left(2 \omega_{0}\right)$. Only in one case level-crossing energy transfer turned out to be of influence.

(iv) The correlation time is composed of several contributions mirroring the phonon-induced rotator transitions.

(v) The influence of the much faster A to E conversion rate at low temperatures is directly observed.

(vi) As expected, the tunnelling frequency of the partially deuterated methyl group is smaller than that of $\mathrm{CH}_{3}$ in the same material.

Comparison of the various molecular crystals in which these observations were made offers an opportunity to study $\mathrm{CH}_{2} \mathrm{D}$ tunnelling for different hindering potentials. In 4-fluoro-toluene the tunnel splitting is large as compared with the NMR frequency.

Upon deuteration the relaxation maximum for $\omega_{\mathrm{t}} \tau_{\mathrm{c}} \approx 1$ disappears as a consequence of the removal of the symmetry coupling between proton spin and rotational levels; level-crossing does not appear in the investigated frequency and temperature range. Both intra- and intermethyl contributions add up to the relaxation peak at $\omega_{0} \tau_{\mathrm{c}}=0.625$. The composition of $\tau_{\mathrm{c}}$ of various contributions analogous to (2) mirrors the correlation spectrum of the nonmagnetic rapid transitions between the rotator states and explains the strange curvature of the $1 / T_{1}$ versus $1 / T$ presentation on the low temperature side. Important and apparently responsible for the relaxation at very low temperatures are the phonon-induced fast $\mathrm{E}$ to $\mathrm{A}$ first order tunnel transitions.

$\mathrm{CHD}_{2}$ tunnelling and relaxation in 4-fluorotoluene behaves rather similarly, but the relaxation strength amounts to only one third of that for $\mathrm{CH}_{2} \mathrm{D}$. This comparison is a way to estimate the importance of intra- and inter-relaxation, since the mechanisms are the same. Small differences in the spectral densities depend on the reduced tunnel splitting.

In 4-chloro-toluene the tunnel splitting in the temperature region under consideration becomes comparable with the NMR frequencies. There is therefore some influence of resonant energy transfer to the rotator system and lattice. For $\mathrm{CH}_{3}$ in the protonated compound (Table 1) the intramolecular relaxation is extremely weak; for $\mathrm{CH}_{2} \mathrm{D} 1 / T_{1}$ is larger, but still too small. Only at very low temperatures the relaxation rates are relatively large because of the availability of spectral densities at suitable values of $\omega_{0} \tau_{\mathrm{c}}$. One 
possible explanation for the anomalous behaviour of 4-chloro-toluene could be an unusually long lifetime of the A level of the rotator ground state which is clearly shortened by the partial deuteration of the $\mathrm{CH}_{3}$ group.

The tunnelling frequency of $\mathrm{CH}_{2} \mathrm{D}$ in 2,6-chlorotoluene in the relevant temperature region is smaller than the proton Zeeman splittings. Quantum effects are therefore less important than for the other compound and for $\mathrm{CH}_{3}$ in the same compound as well. But different from a purely classical behaviour, the correlation time is still governed by at least two apparent activation energies. Since $T_{1}$ is rather long at low temperatures, the $\mathrm{CH}_{2} \mathrm{D}$ tunnel splitting at $5 \mathrm{~K}$ could

[1] Review: D. Cavagnat, J. Chim. Phys. 82, 239 (1985).

[2] W. Press, Single Particle Rotations in Crystals, Springer Tracts in Modern Physics 92, Springer-Verlag, Berlin 1982.

[3] H. Langen, A. S. Montjoie, W. Müller-Warmuth, and H. Stiller, Z. Naturforsch. 42a, 1266 (1987) and references therein.

[4] J. Haupt, Z. Naturforsch. 26a, 1578 (1971).

[5] J. Svare and D. P. Tunstall, J. Phys. C: Solid State Phys. 8, L 559 (1975).

[6] A. E. Zweers and H. B. Brom, Physica 85 b, 223 (1977).

[7] A. E. Zweers, H. B. Brom, and W. J. Huiskamp, Physica 85b, 239 (1977).

[8] H. den Adel, H. B. Brom, W. J. Huiskamp, and B. Alefeld, Physica 112 b, 309 (1982). be measured. It is about 8 times smaller than that of $\mathrm{CH}_{3}$ in the same material.

On the whole, it may be concluded that the various effects observed upon partial deuteration of methyl groups can be explained in terms of the reduced tunnel splitting, of lowering the symmetry, and lifting the restriction in conversion rates.

\section{Acknowledgement}

The financial support of this work by the Deutsche Forschungsgemeinschaft and the Fonds der Chemischen Industrie is gratefully acknowledged.

[9] H. B. Brom, G. Mennenga, D. Cavagnat, and L. Lascombe, J. Phys. C: Solid State Phys. 18, 3407 (1985).

[10] D. Cavagnat, H. Brom, and P. R. Nugteren, J. Chem. Phys. 87, 801 (1987).

[11] K. van Putte, J. Magn. Reson. 2, 216 (1970).

[12] M. Prager, R. Hempelmann, H. Langen, and W. MüllerWarmuth, J. Phys.: Condens. Matter 2, 8625 (1990).

[13] M. Prager, private communication.

[14] H. Langen, W. Müller-Warmuth, and M. Prager, to be published.

[15] G. Vandemale, P. Coppens, and L. van Gerven, Phys. Rev. Lett. 56, 1202 (1986). 